Check for updates

Cite this: Nanoscale Adv., 2021, 3, 3441

Accepted 29th April 2021

DOI: 10.1039/d1na00199

rsc.li/nanoscale-advances

\section{Porous hydrogen-bonded organic framework membranes for high-performance molecular separation $\dagger$}

\author{
Xiao-Tian Jiang, ${ }^{\text {ab }}$ Qi Yin, ${ }^{a}$ Bai-Tong Liu, ${ }^{a}$ Jun-Yu Chen, ${ }^{a}$ Rui Wang ${ }^{a}$ \\ and Tian-Fu Liu (iD *ab
}

\begin{abstract}
Hydrogen-bonded organic frameworks (HOFs) with intrinsic, tunable, and uniform pores are promising candidates to act as membranes for molecular separation, but they are yet to be explored in this field. Herein, a type of HOF membrane based on a thin-film nanocomposite (TFN) membrane containing porous HOF (PFC-1) nanoparticles was successfully fabricated via a facile interfacial polymerization method. The homogeneously distributed HOF nanoparticles can provide direct channels in the polyamide (PA) active layer for molecule separation. Due to the ultrathin nature of the TFN membrane and the highly ordered porous structure of the PFC-1 nanoparticles, these flexible HOF membranes exhibit both ultrahigh water permeability ( $\sim 546.09 \mathrm{~L}$ $\mathrm{m}^{-2} \mathrm{~h}^{-1} \mathrm{bar}^{-1}$ ) and the excellent rejection of dye molecules (e.g., rhodamine $\mathrm{B}$ rejection of $>97.0 \%$ ). Furthermore, long-term operational stability ( $>50 \mathrm{~min}$ ) and satisfactory cycling performance ( $>5$ cycles) have also been achieved. This study may shed light on the fabrication of HOF membranes for liquid-phase molecular separation.
\end{abstract}

The global challenges of water pollution and high energy usage are increasingly supporting the exploitation of membranebased nanotechnology for water purification and wastewater recycling. The established superiority of membrane separation over conventional separation technology lies in its high separation efficiency, low energy requirements, simple operation, high water recovery rates, and environmental friendliness. ${ }^{1}$ Based on a size-exclusion and pressure-driven separation process, nanofiltration (NF) is deemed a promising form of water-purifying nanotechnology, and it has been widely used in water treatment to efficiently reject organic molecules and multivalent ions. ${ }^{2,3}$

As a burgeoning membrane fabrication technology, interfacial polymerization has been widely used to fabricate polyamide

${ }^{a}$ State Key Laboratory of Structural Chemistry, Fujian Institute of Research on the Structure of Matter, Chinese Academy of Sciences, Fujian, Fuzhou 350002, China. E-mail: tfliu@firsm.ac.cn

${ }^{b}$ University of the Chinese Academy of Sciences, Beijing 100049, China

$\dagger$ Electronic supplementary information (ESI) available. See DOI: 10.1039/d1na00199j thin-film composite (TFC) membranes for nanofiltration for water treatment. ${ }^{4}$ TFC membranes are composed of a porous substrate and an ultrathin nanoporous polyamide (PA) active layer formed via an interfacial polymerization (IP) reaction involving monomers. ${ }^{5}$ In previous studies on NF membranes, various functional nanomaterials (e.g., titanium dioxide, silica particles, carbon nanotubes, graphene oxide, zeolites, metalorganic frameworks (MOFs), etc. $)^{6-12}$ were incorporated into the PA active layer to tailor the surface physicochemical properties of the NF membrane (referred to as a thin-film nanocomposite membrane (TFN)) to meet specific water-treatment requirements. ${ }^{13,14}$ In particular, porous nanoparticles can provide efficient transport channels in the dense PA layer which can allow fast molecular transportation and optimize the balance between permeability and rejection. However, some inorganic materials were found to agglomerate in polymeric matrices due to poor compatibility, presenting great challenges for preparing hybrid TFN membranes. Moreover, it is also inevitable that nonselective voids formed in traditional TFN membranes might result in long-term stability issues, especially under high operation pressures. With better affinity for the organic matrix, porous organic framework materials have become a class of outstanding nanofillers for constructing efficient and stable TFN membranes. ${ }^{15-18}$

Self-assembled from organic building blocks through hydrogen bonds and intermolecular interactions, hydrogenbonded organic frameworks (HOFs) $)^{19-21}$ have attracted considerable research attention recently due to their characteristics of high porosity, large surface areas, predictable structures, tunable pore shapes/sizes, solution processability, and easy regeneration. ${ }^{22-25}$ These unique features make HOFs well-suited for TFN membrane fabrication, and the prepared membranes are low-cost and have reclaimable potential. In previous studies, some HOF membranes have been prepared for gas separation, etc. ${ }^{26-29}$ However, maintaining both high porosity and mechanical stability in the case of HOF membranes is still a challenge, and HOF membranes are yet to be applied in the field of liquid purification and separation until now. Differing from other 
inorganic porous nanoparticles, such as zeolites, MOFs, and COFs, HOFs may ameliorate the issues of interfacial defects and poor compatibility within the polymer matrix. In addition, the unique merits of HOFs greatly facilitate the fabrication process and allow for improvements in the separation performance at the atomic level. Therefore, introducing HOFs may provide a novel strategy for constructing robust and high-performance TFN membranes. Learning lessons from previous HOF studies, PFC- 1 is chosen as a nanofiller due to its excellent thermal and chemical stabilities and the mild synthetic conditions needed. ${ }^{30}$ In this study, we report the first example of HOF membranes based on TFN technology. HOF nanoparticles are homogeneously dispersed on a polyethersulfone (PES) substrate and form a continuous membrane with the assistance of the PA active layer. The obtained TFN-based HOF membranes (HOFTFN) exhibit both ultrahigh water permeability and the excellent rejection of dye molecules with long-term operational stability and satisfactory recycling performance, demonstrating promising application potential for molecular separation in liquids.

The crystalline PFC-1 nanoparticles (denoted as Nano-PFC-1) were successfully synthesized via the self-assembly of 1,3,6,8tetrakis ( $p$-benzoic acid)pyrene $\left(\mathrm{H}_{4} \mathrm{TBAPy}\right)$ in a mixed solvent of DMF and $\mathrm{H}_{2} \mathrm{O}$ at room temperature (Fig. 1a, more details in the ESI $\dagger$ ). The addition of $\mathrm{H}_{2} \mathrm{O}$ can increase the nucleation rate of PFC-1, leading to the formation of the desired Nano-PFC-1 particles. The powder X-ray diffraction (PXRD) pattern reveals the high crystallinity and pure phase of Nano-PFC-1 (Fig. 1c). Scanning electron microscopy (SEM) imaging demonstrates that the particle size of Nano-PFC-1 is about $100 \mathrm{~nm} \times 300 \mathrm{~nm}$ (Fig. 1b). Since the water stability of the HOF is an essential factor for HOF membranes used under aqueous conditions, Nano-PFC-1 was exposed to water for 10 days to verify the longterm stability. As shown in Fig. 1c, after being exposed to water for 10 days, the XRD pattern of Nano-PFC-1 remains almost
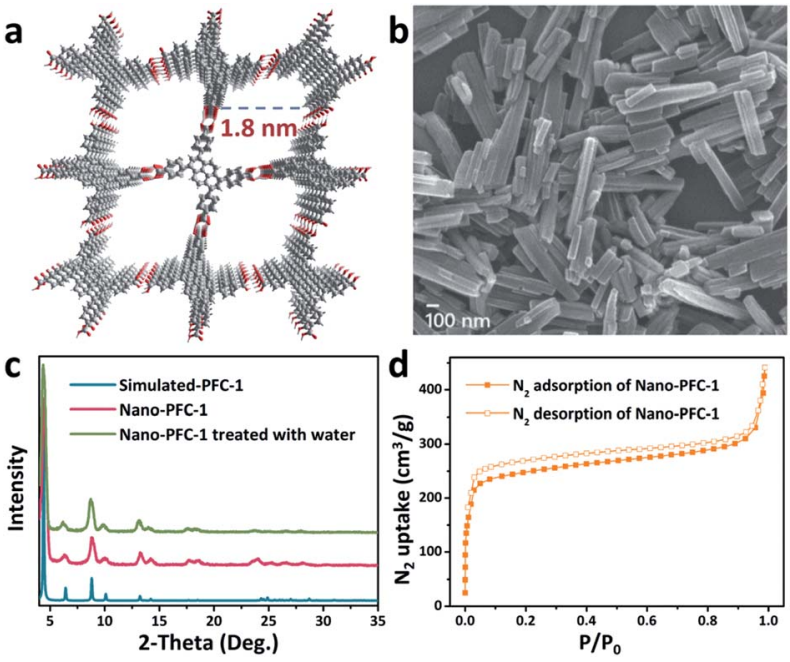

Fig. 1 (a) The two-dimensional porous structure of PFC-1. (b) A scanning electron microscopy (SEM) image, (c) powder XRD patterns, and (d) the $\mathrm{N}_{2}$ adsorption/desorption isotherm (77 K) of Nano-PFC-1. identical to the simulated one, demonstrating the high waterstability of Nano-PFC-1 and its suitability for TFN membrane fabrication for water treatment.

As shown in Scheme 1, Nano-PFC-1 was homogeneously dispersed in an aqueous solution of piperazine (PIP) monomers. Then the mixture was evenly poured onto the surface of a PES substrate and left to stand for $15 \mathrm{~min}$. After removing and evaporating excess solution under atmospheric conditions, the PA/Nano-PFC-1 active layer was formed via an interfacial polymerization method with 1,3,5-benzenetricarbonyl trichloride (TMC), enabling the entrapment of Nano-PFC-1. ${ }^{31,32}$ By using the same method, HOF membranes containing different amounts of Nano-PFC- $1\left(0.1,0.2\right.$, and $\left.0.4 \mathrm{mg} \mathrm{mL}{ }^{-1}\right)$ were successfully fabricated and these were named HOF-TFN-1, HOFTFN-2, and HOF-TFN-4, respectively (Fig. S10 $†$ ).

The homogeneous dispersion of nanoparticles is a key factor determining the uniformity of the obtained HOF-TFN membrane. As shown in Fig. S9, $\dagger$ PIP solution containing Nano-PFC-1 shows an obvious Tyndall effect when irradiated by a laser beam, demonstrating that the nanoparticles are homogeneously dispersed in solution. The good dispersion of NanoPFC-1 in aqueous solution can be attributed to the unbonded polar groups $(-\mathrm{COOH})$ residing on the surfaces of the nanoparticles which are suitably compatible with polar solvents, such as $\mathrm{H}_{2} \mathrm{O}$, based on the idea of "like dissolves like". On the other hand, the partially deprotonated carboxyl groups result in the negatively charged nature of the nanoparticles, with a zeta potential of $-39.58 \mathrm{mV}$ (Fig. S7 $\dagger$ ). Generally, the surface charge significantly influences the dispersity of the nanoparticles and prevents aggregation due to electrostatic repulsion between nanoparticles. $^{33}$ As a result, hydrophilic Nano-PFC-1 is uniformly dispersed into aqueous PIP solution and firmly bonds with the PA layer after reacting with TMC through an interfacial polymerization reaction. A membrane without the addition of Nano-PFC-1 as a nanoporous filler was also fabricated through the same method (named TFC), as a control experiment. These membranes were characterized based on Fourier-transform infrared-diffuse reflectance spectroscopy (FTIR-DRIFTS) (Fig. S6†). Compared with TFC, the HOF-TFN-2 membrane shows an obvious absorption peak at $1708 \mathrm{~cm}^{-1}$, which can be ascribed to the $-\mathrm{COOH}$ stretching vibrations of Nano-PFC-1. The PXRD patterns reveal that the crystal structure of the HOF membrane undergoes no changes during filtration tests (Fig. S8†). ${ }^{31}$ The surface morphologies of the PES substrate, TFC, and HOF-TFN membrane are revealed via both top-view and cross-sectional SEM images (Fig. 2a-f, S1 and $\mathrm{S} 2 \dagger$ ). Compared with TFC, the surface morphology of HOF-TFN is obviously changed. The addition of Nano-PFC-1 makes the surface morphology much rougher, which may improve the surface area of the membrane (Fig. S5 $\dagger$ ) and enhance the water flux. Additionally, upon increasing the amount of Nano-PFC-1, the membranes show a denser and more uniform topography (Fig. S1 $\dagger$ ). This is probably because the introduction of NanoPFC-1 can provide more active sites and therefore anchor more PIP monomers to the substrate surface. The existence of more initial polyamide nuclei then causes even polymerization, resulting in a more compact membrane. As shown in the cross- 


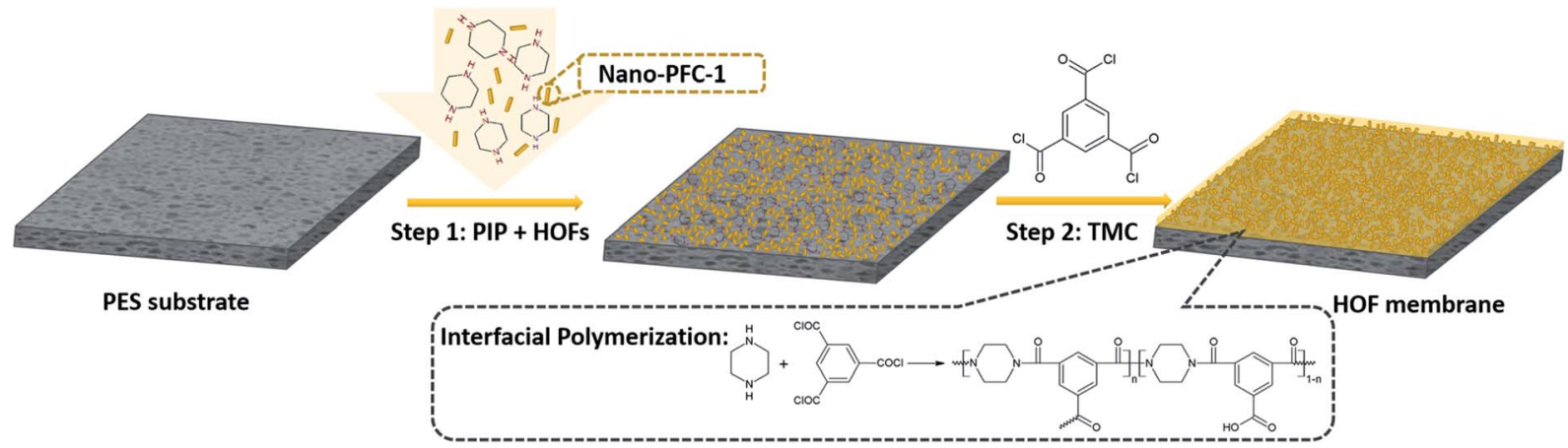

Scheme 1 The preparation process of a HOF-TFN membrane containing PFC-1 nanoparticles via interfacial polymerization.
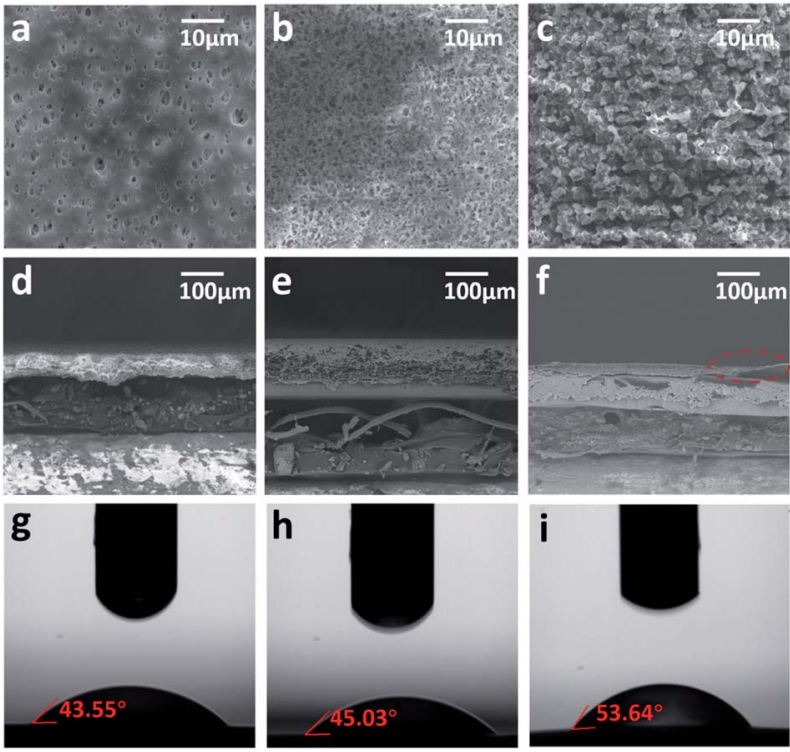

Fig. 2 SEM images showing $(a-c)$ top-down and $(d-f)$ cross-sectional views of ( $a$ and $d$ ) the PES substrate, (b and e) TFC, and (c and f) HOFTFN-2. Water-in-air contact angles for PES (g), TFC (h), and HOF-TFN2 (i)

section images in Fig. 2 and $\mathrm{S} 1, \dagger$ every HOF-TFN membrane contains a porous PES substrate tightly covered with a dense polyamide PA layer. The thickness of the polyamide/Nano-PFC1 layer was about $7 \mu \mathrm{m}$ (Fig. $\mathrm{S} 2 \dagger$ ).

The surface hydrophilicity is another considerable factor relating to nanofiltration membranes and it is highly connected to the water permeability. ${ }^{34}$ As shown in Fig. $2 \mathrm{~g}-\mathrm{i}$ and S2 and $\mathrm{S} 3, \dagger$, compared with the TFC membrane, the contact angle slightly increases for HOF-TFN due to the incorporation of Nano-PFC-1. The transient water contact angle of the TFC membrane is $45.03^{\circ}$. Upon increasing the amount of Nano-PFC1 , the contact angle increases to $49.93^{\circ} \pm 0.5^{\circ}$ for HOF-TFN- 1 , $53.64^{\circ} \pm 0.5^{\circ}$ for HOF-TFN-2, and $61.7 \pm 0.5^{\circ}$ for HOF-TFN-4 (Table S1 $\dagger$ ). This result further demonstrates that the incorporation of Nano-PFC-1 does not greatly change the hydrophilicity of the membrane.

To explore the capacity for liquid separation, the water permeance of the HOF-TFN membranes was first evaluated. The permeance (P) values, in $\mathrm{L} \mathrm{m}^{-2} \mathrm{~h}^{-1} \mathrm{bar}^{-1}$, were calculated using the following equation:

$$
P=\frac{V}{A \times t \times \Delta p}
$$

where $V(\mathrm{~L})$ is the volume of permeate water during a given time $t$ (h) using a membrane effective filtration area $A\left(\mathrm{~m}^{2}\right)$ at a certain operating pressure $\Delta p$ (bar). The water permeance performances of the HOF-TFN membranes are shown in Fig. 3a. Compared with the pure TFC membrane, the HOF-TFN membranes show obviously improved water flux levels. Among them, HOF-TFN-2 shows the highest water permeability of $546.09 \mathrm{~L} \mathrm{~m}^{-2} \mathrm{~h}^{-1}$ bar $^{-1}\left(153.87 \mathrm{~L} \mathrm{~m}^{-2} \mathrm{~h}^{-1} \mathrm{bar}^{-1}\right.$ for TFC and $202.94 \mathrm{~L} \mathrm{~m}^{-2} \mathrm{~h}^{-1} \mathrm{bar}^{-1}$ for HOF-TFN-1). Upon further increasing the Nano-PFC-1 amount to $0.4 \mathrm{mg} \mathrm{mL}^{-1}$, the permeability dropped to $469.63 \mathrm{~L} \mathrm{~m}^{-2} \mathrm{~h}^{-1} \mathrm{bar}^{-1}$. As we know, the water permeability of HOF membranes is related to many factors, including the molecular structure and size-sieving effects, the intrinsic diffusion coefficient, interactions involving the membrane surface and pores, and so on. ${ }^{35}$ The aperture size of Nano-PFC-1 is about $1.8 \mathrm{~nm}$, which is larger
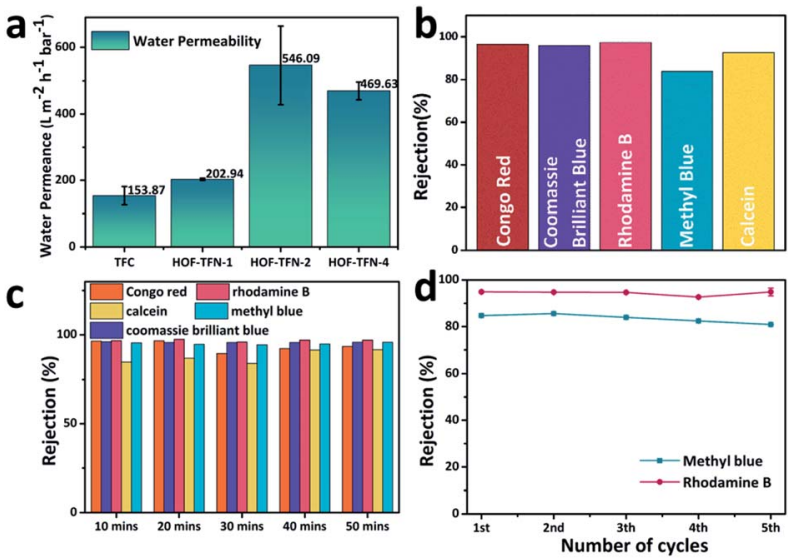

Fig. 3 (a) The water permeance values of the TFC, HOF-TFN-1, HOFTFN-2, and HOF-TFN-4 membranes. (b) The different dye rejection rates of HOF-TFN-2 in a short time (about $5 \mathrm{~min}$ ). (c) The dye separation performances of the HOF-TFN-2 membrane during 50 min of testing. (d) The consistent dye rejection performance of the HOF-TFN2 membrane over 5 reuse cycles. 
than that of water molecules $(0.28 \mathrm{~nm})$. The highly ordered porosity of the Nano-PFC-1 structure in the HOF-TFN membranes could provide additional pathways for $\mathrm{H}_{2} \mathrm{O}$ molecules and lead to higher water permeance. In addition, voids between Nano-PFC-1 and the polymer could also increase the water flux. $^{32}$ However, the introduction of excess Nano-PFC-1 to the TFN-based membrane may inevitably encourage the uneven distribution of nanoparticles on the active layer. The formation of a partially inhomogeneous surface structure would result in a decrease in hydrophilicity and water flux. Based on all the factors above, the use of an optimized amount of Nano-PFC-1 can result in a HOF-TFN membrane with outstanding water permeance in contrast to the TFC membrane alone. The water permeance values at different pressures are also important for certain special applications. A series of pressure-driven operations (under 0.5 bar, 1.0 bar, 2.0 bar, 3.0 bar, and 4.0 bar pressure) was used to assess the stability of the HOF membranes, and the results are summarized in Fig. S11. $\dagger$ Taking the HOF-TFN-2 membrane as an example, the membrane exhibited high water permeance values of $509.05 \mathrm{~L}$ $\mathrm{m}^{-2} \mathrm{~h}^{-1}$ bar $^{-1}$ under 0.5 bar; $569.09 \mathrm{~L} \mathrm{~m}^{-2} \mathrm{~h}^{-1}$ bar $^{-1}$ under 1.0 bar; $518.75 \mathrm{~L} \mathrm{~m}^{-2} \mathrm{~h}^{-1}$ bar $^{-1}$ under 2.0 bar; $538.49 \mathrm{~L} \mathrm{~m}^{-2} \mathrm{~h}^{-1}$ bar $^{-1}$ under 3.0 bar; and $494.54 \mathrm{~L} \mathrm{~m}^{-2} \mathrm{~h}^{-1}$ bar $^{-1}$ under $4.0 \mathrm{bar}$, confirming that it shows the required durability and mechanical robustness.

To further explore the nanofiltration performance of HOFTFN membranes for removing divalent salts and hazardous organic dye molecules from industrial wastewater, we conducted experiments to evaluate the separation abilities toward $\mathrm{Na}_{2} \mathrm{SO}_{4}$ and five toxic dyes with different molecular weights and sizes (methyl blue (MB), $799 \mathrm{~g} \mathrm{~mol}^{-1}$, skin irritant; rhodamine B (RB), $479 \mathrm{~g} \mathrm{~mol}^{-1}$, damaging to the eyes and throat; Congo red (CR), $686 \mathrm{~g} \mathrm{~mol}^{-1}$, carcinogen and mutagen; calcein (CC), $623 \mathrm{~g}$ $\mathrm{mol}^{-1}$, respiratory tract irritant; and Coomassie brilliant blue
(CB), $826 \mathrm{~g} \mathrm{~mol}^{-1}$, epidermal cell irritant; Fig. S12†). Herein, the $\mathrm{SO}_{4}{ }^{2-}$ concentration was measured via ion chromatography. The dye concentrations in the feed and permeate filtered through the HOF-TFN membranes were calculated based on UV-vis spectroscopy absorbance (Fig. 4 and S14-S16†). The rejection rate $(R, \%)$ values for the solutions were determined using the following equation:

$$
R=\frac{C_{\mathrm{f}}-C_{\mathrm{p}}}{C_{\mathrm{f}}} \times 100 \%
$$

where $C_{\mathrm{f}}$ and $C_{\mathrm{p}}$ are the dye concentrations in the original and filtered solutions, respectively. ${ }^{32}$ As shown in Fig. $\mathrm{S} 13, \uparrow$ the HOF membranes generally show certain divalent salt retention abilities due to the densely formed PA/Nano-PFC-1 layer and assembled interfacial paths. Taking the HOF-TFN-2 membrane as an example, we tested the dye separation capacities. The retention results are summarized in Fig. $3 \mathrm{~b}$ and $514 . \dagger$ The membrane shows excellent rejection performance toward all dyes (95.85\% for CR, $96.47 \%$ for CB, $97.26 \%$ for RB, $83.70 \%$ for $\mathrm{MB}$, and $92.59 \%$ for $\mathrm{CC}$ in about $5 \mathrm{~min}$ ), confirming that it meets the basic dye separation requirements.

Furthermore, the separation stability of the HOF-TFN-2 membrane was evaluated based on long-term continuous experiments. As shown in Fig. 4 and $\mathrm{S} 15, \dagger$ the absorbance peak of the dye in solution significantly decreases after filtration, showing the excellent dye separation abilities. During the filtration process, there are only slightly decreases in dye retention (Fig. $3 \mathrm{c}$ and $\mathrm{S} 15 \mathrm{c} \dagger$ ), confirming the reliable retention efficiency of the HOF-TFN-2 membrane for at least 50 minutes and even 300 minutes.

Finally, the cycling durability of the HOF-TFN-2 membrane was also evaluated based on a 5-cycle nanofiltration test involving methyl blue and rhodamine $\mathrm{B}$ rejection, separately. Each cycle consists of dye solution filtration through the HOF-
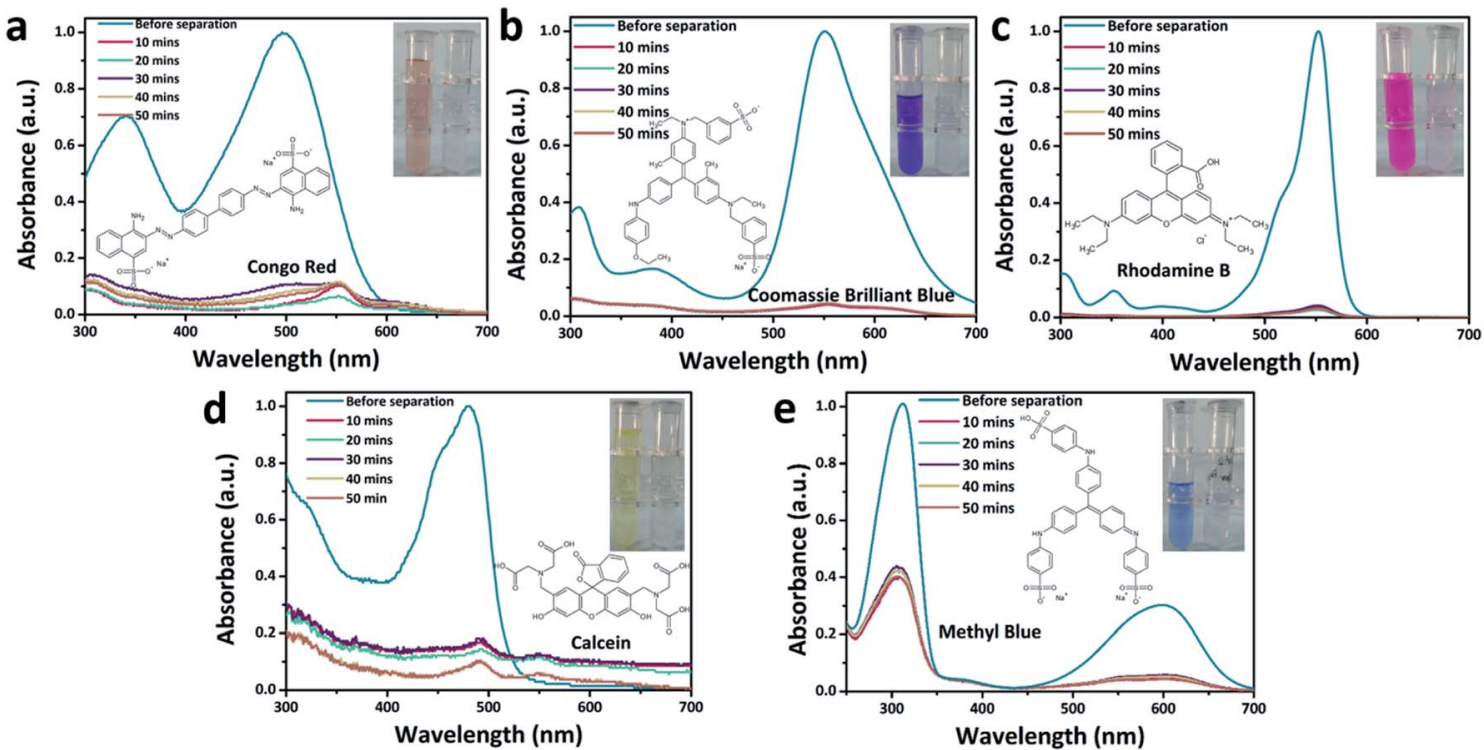

Fig. 4 The chemical structures of the dyes, digital photos, and UV-vis spectra of the feed (blue lines) and permeate (lines in other colors) solutions: (a) Congo red, (b) Coomassie brilliant blue, (c) rhodamine B, (d) calcein, and (e) methyl blue. 
TFN-2 membrane for $1 \mathrm{~h}$ followed by $\mathrm{H}_{2} \mathrm{O}$ filtration for $30 \mathrm{~min}$. As shown in Fig. 3d and S16, $\dagger$ even after 5 cycles of operation, the HOF-TFN-2 membrane shows consistent dye rejection performance, thus confirming the desired long-term retention durability and mechanical pore robustness. To sum up, the obtained HOF-TFN-2 membrane with high dye retention and water permeation flux is an excellent candidate for wastewater treatment.

\section{Conclusions}

In summary, we exploited a facile interfacial polymerization strategy to fabricate TFN-based HOF membranes using a PA/ Nano-PFC-1 active layer on a PES substrate. This strategy is proved to load HOF nanoparticles at a controlled concentration and with an even distribution on the active layer, and the fabrication of the HOF membranes can be completed in a short time. Due to the ultrathin active layer and the uniform pore structure of the HOF, the prepared membranes exhibited ultrahigh water-permeability $\left(\sim 546.09 \mathrm{~L} \mathrm{~m}^{-2} \mathrm{~h}^{-1} \mathrm{bar}^{-1}\right)$ and the excellent rejection of organic dye molecules. Accordingly, the membranes exhibited long-term operational stability and satisfactory recyclability. We think that this cost-effective IP strategy opens up a new pathway for the design and fabrication of HOF membranes and it can expand the application field of HOF materials.

\section{Conflicts of interest}

The authors declare no conflicts of interest.

\section{Acknowledgements}

We gratefully acknowledge the National Key Research and Development Program of China (Grant No. 2018YFA0208600), CAS-Iranian Vice presidency for Science and Technology Joint Research Project (Grant No. 121835KYSB20200034), National Natural Science Foundation of China (Grant No. 21871267, 22071246, 22005305), and China Postdoctoral Science Foundation (Grant No. 2020M671955).

\section{Notes and references}

1 R. Zhang, Y. Liu, M. He, Y. Su, X. Zhao, M. Elimelech and Z. Jiang, Chem. Soc. Rev., 2016, 45, 5888-5924.

2 M. R. Esfahani, S. A. Aktij, Z. Dabaghian, M. D. Firouzjaei, A. Rahimpour, J. Eke, I. C. Escobar, M. Abolhassani, L. F. Greenlee and A. R. Esfahani, Sep. Purif. Technol., 2019, 213, 465-499.

3 A. W. Mohammad, Y. Teow, W. Ang, Y. Chung, D. OatleyRadcliffe and N. Hilal, Desalination, 2015, 356, 226-254.

4 M. B. M. Y. Ang, J. M. Pereira, C. A. Trilles, R. R. Aquino, S.-H. Huang, K.-R. Lee and J. Y. Lai, Sep. Purif. Technol., 2019, 210, 521-529.

5 F. Zhang, J. B. Fan and S. Wang, Angew. Chem., Int. Ed., 2020, 59, 21840-21856.
6 J. Wang, Y. Wang, J. Zhu, Y. Zhang, J. Liu and B. Van der Bruggen, J. Membr. Sci., 2017, 533, 279-288.

7 Y. L. Li, I. A. Kinloch and A. H. Windle, Science, 2004, 304, 276-278.

8 C. S. Rodolfo, I. Kazuo, M. Jun, S. Shigeru, M. G. Aaron, A. Celia, T. Yoshihiro, A. Yamanaka, S. Tejiima and K. Fujisawa, Nanoscale, 2020, 12, 19628-19637.

9 S. Zinadini, A. A. Zinatizadeh, M. Rahimi, V. Vatanpour and H. Zangeneh, J. Membr. Sci., 2014, 453, 292-301.

10 L. Yang, Z. Wang and J. Zhang, J. Membr. Sci., 2017, 532, 7686.

11 Z. Hu, Y. Wang and D. Zhao, Chem. Soc. Rev., 2021, 50, 46294683.

12 J. Hou, P. D. Sutrisna, Y. Zhang and V. Chen, Angew. Chem., Int. Ed., 2016, 55, 3947-3951.

13 J. R. Werber, C. O. Osuji and M. Elimelech, Nat. Rev. Mater., 2016, 1, 1-15.

14 N. A. Khan, R. Zhang, H. Wu, J. Shen, J. Yuan, C. Fan, L. Cao, M. A. Olson and Z. Jiang, J. Am. Chem. Soc., 2020, 142, 1345013458.

15 S. Sorribas, P. Gorgojo, C. Téllez, J. Coronas and A. G. Livingston, J. Am. Chem. Soc., 2013, 135, 15201-15208.

16 J. Guo, Y. Zhang, Y. Zhu, C. Long, M. Zhao, M. He, X. Zhang, J. Lv, B. Han and Z. Tang, Angew. Chem., Int. Ed., 2018, 57, 6873-6877.

17 Z. Wang, S. Zhang, Y. Chen, Z. Zhang and S. Ma, Chem. Soc. Rev., 2020, 49, 708-735.

18 J. Campbell, R. Davies, D. C. Braddock and A. Livingston, J. Mater. Chem. A, 2015, 3, 9668-9674.

19 T. Li, B. T. Liu, Z. B. Fang, Q. Yin and T. F. Liu, J. Mater. Chem. A, 2021, 9, 4687-4691.

20 A. Karmakar, R. Illathvalappil, B. Anothumakkool, A. Sen, P. Samanta, A. V. Desai, S. Kurungot and S. K. Ghosh, Angew. Chem., Int. Ed., 2016, 55, 10667-10671.

21 J. Lü and R. Cao, Angew. Chem., Int. Ed., 2016, 55, 9474-9480.

22 Z. B. Han, Z. Z. Xiao, M. Hao, D. Q. Yuan, L. Liu, N. Wei, H. M. Yao and M. Zhou, Cryst. Growth Des., 2015, 15, 531533.

23 Y. He, S. Xiang and B. Chen, J. Am. Chem. Soc., 2011, 133, 14570-14573.

24 F. Hu, C. Liu, M. Wu, J. Pang, F. Jiang, D. Yuan and M. Hong, Angew. Chem., Int. Ed., 2017, 56, 2101-2104.

25 R. B. Lin, Y. He, P. Li, H. Wang, W. Zhou and B. Chen, Chem. Soc. Rev., 2019, 48, 1362-1389.

26 J. F. Feng, T. F. Liu and R. Cao, Angew. Chem., Int. Ed., 2020, 59, 22392-22396.

27 K. Ma, P. Li, J. H. Xin, Y. Chen, Z. Chen, S. Goswami, X. Liu, S. Kato, H. Chen, X. Zhang, J. Bai, M. C. Wasson, R. R. Maldonado, R. Q. Snurr and O. K. Farha, Cell Rep. Phys. Sci., 2020, 1, 100024.

28 S. Feng, Y. Shang, Z. Wang, Z. Kang, R. Wang, J. Jiang, L. Fan, W. Fan, Z. Liu, G. Kong, Y. Feng, S. Hu, H. Guo and D. Sun, Angew. Chem., Int. Ed., 2020, 59, 3840-3845.

29 B. T. Liu, X. H. Pan, D. Y. Nie, X. J. Hu, E. P. Liu and T. F. Liu, Adv. Mater., 2020, 32, e2005912.

30 Q. Yin, P. Zhao, R. J. Sa, G. C. Chen, J. Lu, T. F. Liu and R. Cao, Angew. Chem., Int. Ed., 2018, 57, 7691-7696. 
31 F. Xiao, X. Hu, Y. Chen and Y. Zhang, ACS Appl. Mater. Interfaces, 2019, 11, 47390-47403.

32 J. Zhu, L. Qin, A. Uliana, J. Hou, J. Wang, Y. Zhang, X. Li, S. Yuan, J. Li, M. Tian, J. Lin and B. Van der Bruggen, ACS Appl. Mater. Interfaces, 2017, 9, 1975-1986.
33 J. Hou, C. Bao, S. Qu, X. Hu, S. Nair and Y. Chen, Appl. Surf. Sci., 2018, 459, 185-193.

34 H. Shen, S. Wang, H. Xu, Y. Zhou and C. Gao, J. Membr. Sci., 2018, 565, 145-156.

35 J. M. Gohil and P. Ray, Sep. Purif. Technol., 2017, 181, 159182. 